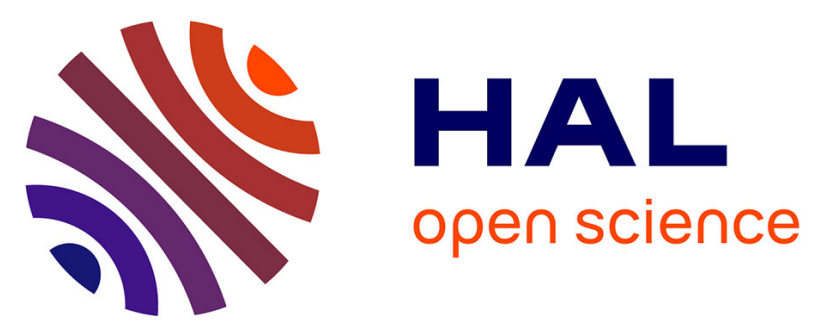

\title{
Using Doppler shift induced by Galvanometric mirror scanning to reach shot noise limit with laser optical feedback imaging setup
}

O Jacquin, E Lacot, O Hugon, H Guillet de Chatelus

\section{- To cite this version:}

O Jacquin, E Lacot, O Hugon, H Guillet de Chatelus. Using Doppler shift induced by Galvanometric mirror scanning to reach shot noise limit with laser optical feedback imaging setup. Applied optics, 2015, 54 (8), pp.1978-1983. 10.1364/AO.54.001978 . hal-01130641

\section{HAL Id: hal-01130641 \\ https://hal.science/hal-01130641}

Submitted on 13 Mar 2015

HAL is a multi-disciplinary open access archive for the deposit and dissemination of scientific research documents, whether they are published or not. The documents may come from teaching and research institutions in France or abroad, or from public or private research centers.
L'archive ouverte pluridisciplinaire HAL, est destinée au dépôt et à la diffusion de documents scientifiques de niveau recherche, publiés ou non, émanant des établissements d'enseignement et de recherche français ou étrangers, des laboratoires publics ou privés. 


\title{
Using Doppler shift induced by galvanometric mirrors scanning to reach the shot noise limit with the laser optical feedback imaging (LOFI) setup
}

\author{
O. Jacquin ${ }^{* 1}$, E. Lacot ${ }^{1}$, O. Hugon ${ }^{1}$, and H. Guillet de Chatelus ${ }^{2}$ \\ ${ }^{1}$ Univ. Grenoble Alpes, LIPHY, F-38000 Grenoble, France \\ ${ }^{2} \mathrm{CNRS}$, LIPHY, F-38000 Grenoble, France \\ *Corresponding author: Olivier.jacquin@ujf-grenoble.fr
}

\begin{abstract}
We propose a new method to remove the contribution of parasitic reflections in the images of laser optical feedback imaging technique. This method is very simply and allows to extend LOFI technique to "long distance" application, as imaging through a fog or a smoke. The LOFI technique is an ultrasensitive imaging technique very interesting for imaging objects through a scattering medium. However, the LOFI sensitivity can be dramatically limited by parasitic optical feedback occurring in the experimental setup. In previous papers [11, 12], we have already proposed methods to filter a parasitic optical feedback but they are not well suited to metric working distances. This is why we propose a new method using a Doppler frequency shift induced by the moving mirror which allows to scan the object to image. This Doppler frequency shift allows to distinguish the photons reflected by the target and the parasitic photons reflected by the optical components in the experimental setup. In this paper, we demonstrate theoretically and experimentally the possibility to filter the parasitic reflection in LOFI images by using this Doppler frequency shift. With this method, the signal to noise ratio is significantly improved (by a factor 15) and we obtain a shot noise limited image through a scattering medium of an object at 3 meters from the detector.
\end{abstract}

Key word: Imaging systems; Interferometric imaging; Imaging through turbid media

\section{INTRODUCTION}

Imaging objects through or embedded within a scattering media has always been a major issue linked to many applications such as imaging through biological tissues or imaging through the fog. The main challenge is to realize images through a turbid medium with both a high resolution and a good signal-to-noise ratio (SNR). The information needed to obtain diffraction limited images is carried by ballistic photons. However, compared to scattered photons, their number rapidly decreases with depth, which dramatically reduces the SNR. Consequently, imaging through scattering media with diffraction-limited resolution requires both the detection of the ballistic photons and the rejection of the scattered light. The filtering of the scattered light is usually carried out by limiting the depth of focus of the imaging setup. Efficient methods for accomplishing this goal include confocal technique [1], time-resolved techniques [2], and optical coherent technique [3]. However, the thickness of the scattering medium explored with these techniques is limited to about 20 mean free paths, and these methods require a high optical power in the medium to compensate for the losses in ballistic photons, which is generally not compatible with "eyes safety conditions" because viewing of the diffuse reflection may be dangerous close to the laser for the operator or the people around. In [4] we demonstrated that the laser optical feedback imaging (LOFI) technique is well suited for imaging through scattering media with lower optical power than the method previously cited.

LOFI is an ultrasensitive laser autodyne interferometer and also a confocal imaging technique combining the great accuracy of optical interferometry with the very high sensitivity of class B lasers to optical feedback [5]. In this technique the laser is both source and detector. The detection limit is then given by the quantum noise of the laser as demonstrated in [6], in consequence this technique is shot noise limited. In addition, the system is self-aligned and simple since there is no external reference arm as in traditional heterodyne interferometers. For these reasons the LOFI technique is used for many applications as vibrometry [7], velocimetry [8], near field measurement [9], imaging through scattering media [10]. The LOFI technique is used more particularity for applications which require extreme optical sensitivity or shot noise limited detection [6] and low optical power compatible with eye safety or medical application [4]. However, parasitic optical feedbacks due to unwanted reflections in the experimental setup dramatically affect the sensitivity of the technique. In [11, 12] we proposed different solutions to remove parasitic optical feedbacks and reach shot noise limited detection. In [11] the solution was based on a two- 
beam optical setup with an overlap of two beams on the target which is more difficult to realize when the working distance increases. In [12] we used the acoustic tagging close to the studied target. These methods are well suited for applications with millimetric working distances but no for metric working distances as imaging through the fog. In this paper, we propose a new method suitable for the metric working distances applications as imaging through the fog in eyes safety conditions (low optical power). This method utilizes the Doppler frequency shift caused by a moving mirror used to scan the target. This Doppler frequency shift allows to distinguish the photons reflected by the target and the parasitic photons reflected by the optical components in the experimental setup.

\section{LOFI SETUP FOR METRIC WORKING DISTANCE APPLICATIONS}

A scheme of our experimental setup is shown in figure 1. The laser is a $\mathrm{CW} \mathrm{Nd}^{3+}$ :YAG microchip with an output power $\mathrm{P}_{\text {out }}=10 \mathrm{~mW}$, at the wavelength $\lambda=1064 \mathrm{~nm}$. This laser has a relaxation frequency $F_{R}$ in the megahertz range. The laser beam is frequency shifted through acousto-optic modulators (AOM). The frequency shift is tunable and is denoted by $\mathrm{F}_{\mathrm{e}} / 2$. Then the laser beam is focalized onto the studied target using a telescope and the back-reflected light is reinjected in the laser by the same path. After a round-trip of the light, the frequency shift introduced by the AOMs is $\mathrm{F}_{\mathrm{e}}$. The photons re-injected inside the laser create an optical beating and lead to a modulation of the laser output power at the frequency $\mathrm{F}_{\mathrm{e}}$. There is a resonant amplification of this modulation if $\mathrm{F}_{\mathrm{e}}$ is close to the laser relaxation frequency $\mathrm{F}_{\mathrm{R}}$ and in this case, the feedback detection is a priori shot noise limited. A beam splitter sends a fraction of the output power on the photodiode connected to a lock-in amplifier which gives the amplitude and phase of the output power modulation (LOFI signal). The photodiode is also connected to an oscilloscope with Fast Fourier transform (FFT) application to study the RF spectrum of the output power modulation. The LOFI images (amplitude and phase) are obtained pixel by pixel (i.e. point by point, line after line). In our setup, the target is imaged using a single axis galvanometric mirror scanner coupled with a motorized rotation stage for the second axis.

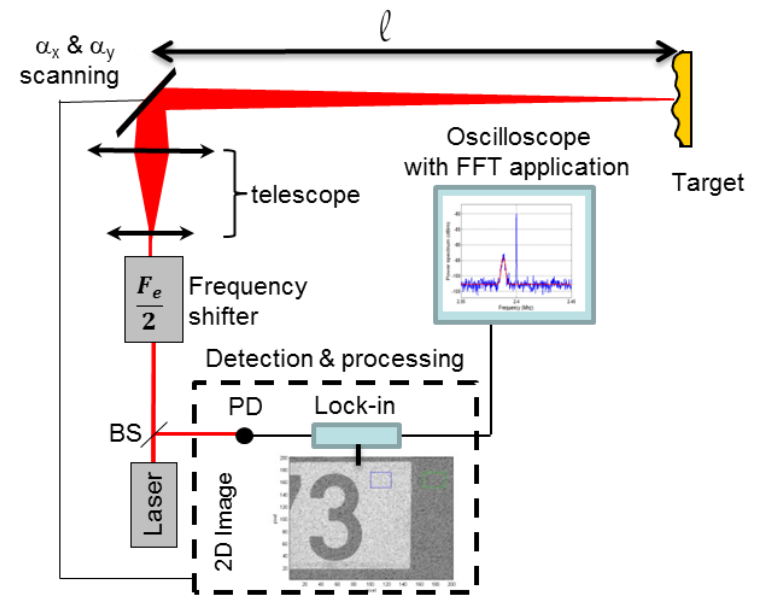

Figure 1: Schematic diagram of LOFI setup for long distance imaging. $\mathrm{PD}$, photodiode; BS, beam splitter.

A feature of this "long distance" setup (metric working distance) is that the scanner is situated after the focalization optics. The rotation of the galvanometric mirror is continuous and the typical angular speed $(\dot{\alpha})$ is of order of $1 \mathrm{rad} . \mathrm{s}^{-1}$. When the laser beam is misaligned with the galvanometric mirror axis, a broadband Doppler frequency shift appears.

\section{DOPPLER EFFECTS CALCULATION FOR PUNCTUAL SCATTERER}

We propose to determine the effect of the scanning on the LOFI signal when the target is a punctual scatterer. Indeed, when the laser beam is misaligned with the rotation axis of the moving mirror then the distance between the laser and the target changes during the scanning which induces a Doppler frequency shift. When the laser beam propagation axis and the rotation axis are aligned, the distance between the laser and the target also changes but this variation is symmetrical which induces Doppler frequency broadening (but no Doppler frequency shift). We propose to determine the dependency of these Doppler effects with the optogeometrical parameters of the LOFI setup. For this we consider a punctual target which is scanned by a Gaussian laser beam using a rotating mirror. The scanner is composed by a galvanometric mirror for horizontal scanning (fast axis) which it is mounted on a step motor for vertical scanning (slow axis). In the fast axis, the mirror reaches very quickly the scanning speed and we can consider a continuous rotation with a constant speed. The Doppler effects are caused by the fast scanning. To determine these effects (frequency shift and frequency broadening) in the horizontal direction, we work in the virtual space (fig.2) and we consider only a bidimensional problem (X and $\mathrm{Z}$ directions) in order to simplify calculus. The figure 2 shows a target and the laser beam in real and virtual spaces.

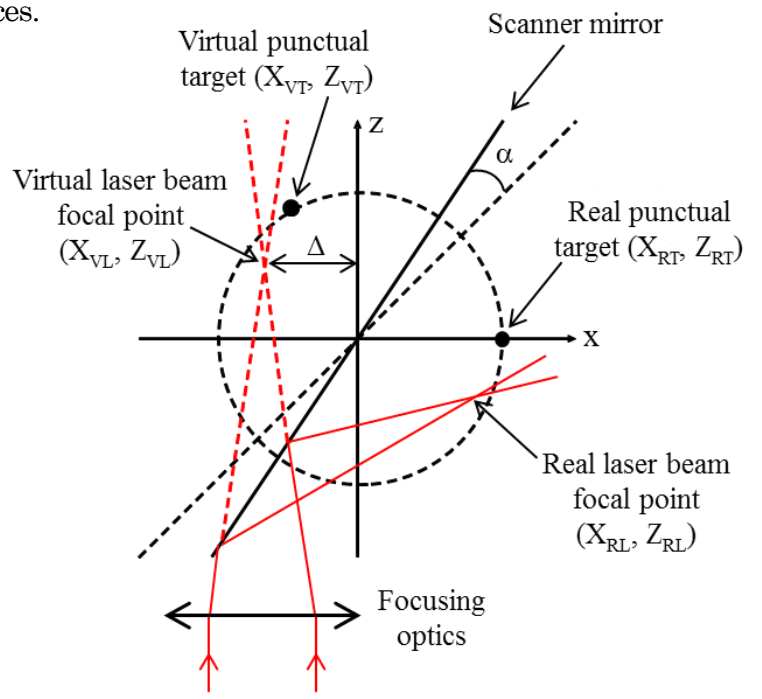

Figure $2:$ scanning in real and virtual spaces. The focused laser beam is deflected by the galvanometric mirror to scan the target. When $\alpha=0$ the mirror makes an angle of $45^{\circ}$ with $\mathrm{x}$ and $\mathrm{z}$ axis. $\Delta$ is the distance between the propagation direction of the Gaussian laser beam and the rotation axis of the galvanometric mirror. The rotation axis of the galvanometric mirror is at the origin of the cartesian coordinate system $(x=0, z=0)$. In the real space, the laser beam focal point is situated at $X_{R L}$ and $Z_{R L}$, and the punctual target is situated at $X_{R T}=\ell$ and $Z_{R T}=0$. In the virtual space, the laser beam focal point is situated at $X_{V L}=\Delta$ and, $Z_{V L}=\mathrm{L}$ and the virtual punctual target is situated at $X_{V T}=-\ell \sin (2 \alpha)$ and $Z_{V T}=\ell \cos (2 \alpha)$. 
In the virtual space, the gaussian beam is fixed and it is the target which moves during the scan. The target moves along the dotted circle (see Fig.2). The laser beam presents a slight misalignment $(\Delta)$ with the rotation axis of the galvanometric mirror. In this space, the expression of the laser beam amplitude which illuminates the punctual target of the figure 2 is given by:

$$
\begin{gathered}
E_{\text {Laser }}\left(X_{V T}-X_{V L}, Z_{V T}-Z_{V L}\right)=\frac{E_{0} W_{0}}{W\left(Z_{V T}-Z_{V L}\right)} \\
\times \exp \left(-\frac{\left(X_{V T}-X_{V L}\right)^{2}}{W\left(Z_{V T}-Z_{V L}\right)^{2}}\right) \exp \left(j \frac{\pi}{\lambda} \frac{\left(X_{V T}-X_{V L}\right)^{2}}{R\left(Z_{V T}-Z_{V L}\right)}\right) \\
\times \exp \left(j \frac{2 \pi}{\lambda}\left(Z_{V T}-Z_{V L}\right)\right) \\
W(Z)=W_{0} \sqrt{1+\frac{Z^{2}}{Z_{R}^{2}}}
\end{gathered}
$$$$
\text { With : } \quad R(Z)=Z\left(1+\frac{Z_{R}^{2}}{Z^{2}}\right)
$$$$
Z_{R}=\frac{\pi W_{0}^{2}}{\lambda}
$$

$\mathrm{W}_{0}$ is the waist of the gaussian beam at the focal point and $\lambda$ is the laser wavelength. $E_{0}$ is the amplitude of the laser beam at $X=X_{V L}$ and $Z=Z_{V L}$ (focal point).

With the assumption of small rotation angles for the scanning mirror and by considering only the term with $\alpha$ and $\alpha^{2}$, the previous equation becomes:

$$
\begin{aligned}
& E_{\text {Laser }}(\alpha)=E_{0} \frac{W_{0}}{W(\ell-L)} \exp \left(-\frac{(\ell 2 \alpha-\Delta)^{2}}{W(\ell-L)^{2}}\right) \\
& \times \exp \left(j \frac{2 \pi}{\lambda}\left(\ell-L-\ell 2 \alpha^{2}\right)\right) \exp \left(j \frac{\pi}{\lambda} \frac{(\ell 2 \alpha-\Delta)^{2}}{R(\ell-L)}\right)
\end{aligned}
$$

Furthermore, we consider the case where the beam is focalised very near the target:

$W(\ell-L) \approx W_{0}$ and $R(\ell-L) \approx \frac{Z_{R}^{2}}{(\ell-L)} \approx \infty$

In this case, the laser beam amplitude on the punctual target is simply given by:

$$
\begin{aligned}
& E_{\text {Laser }}(\alpha)=E_{0} \exp \left(-\frac{(\ell 2 \alpha-\Delta)^{2}}{W_{0}^{2}}\right) \\
& \times \exp \left(j \frac{2 \pi}{\lambda}\left(\ell-L-\ell 2 \alpha^{2}\right)\right)
\end{aligned}
$$

The punctual target reflects a part of the laser beam which is reinjected in the laser. In this case, the LOFI signal can be written [13]:

$$
\begin{aligned}
& S(\alpha, t) \propto \operatorname{Re}\left\{G_{l o f i} \sqrt{R_{t}}\left[\exp \left(-\frac{(\ell 2 \alpha-\Delta)^{2}}{W_{0}^{2}}\right)\right]^{2}\right. \\
& \left.\times\left[\exp \left(j \frac{2 \pi}{\lambda}\left(\ell-L-\ell 2 \alpha^{2}\right)\right)\right]^{2} \exp \left(-j 2 \pi F_{e} t\right)\right\}
\end{aligned}
$$

As demonstrated in [13], the square corresponds to symmetrical coupling between the object and the electric field. Glofi is a gain factor caused by the laser dynamics. $R_{t}$ corresponds to the power effective reflectivity of the punctual target.

To quantify the Doppler effects, we must take into account the moving mirror in the LOFI signal. We assume a constant rotation speed for the mirror. At the time t, we have $\alpha=\dot{\alpha}$. We

define a novel origin for time: $T=t-\frac{\Delta}{2 \ell \dot{\alpha}}=\frac{\alpha}{\dot{\alpha}}-\frac{\Delta}{2 \ell \dot{\alpha}}$

In the virtual space, the target of the figure 2 is situated at $X_{V T}=\Delta$ at the time $\mathrm{T}=0$. By neglecting the constant term, the equation 3 becomes:

$$
\begin{aligned}
& S(T) \propto \operatorname{Re}\left\{G_{l o f i} \sqrt{R_{t}}\left[\exp \left(-(\ell 2 \dot{\alpha} T)^{2}\left(\frac{2}{W_{0}^{2}}+j \frac{2 \pi}{\lambda \ell}\right)\right)\right]\right. \\
& \left.\times \exp \left(-j 2 \pi\left(F_{e}+4 \frac{\Delta}{\lambda} \dot{\alpha}\right) T\right)\right\}
\end{aligned}
$$

The equation 5 shows that the frequency of the LOFI signal is shifted, and that the optical beating is is now at the frequency $\mathrm{F}_{\mathrm{e}}+\mathrm{F}_{\text {dop }}$, with:

$$
F_{\text {dop }}=4 \frac{\Delta}{\lambda} \dot{\alpha}
$$

Eq. 6 shows that the Doppler frequency shift depends on the misalignment $\Delta$ and on the mirror rotation speed $\dot{\alpha}$. We can therefore adjust $\mathrm{F}_{\text {dop }}$ by translating the mirror or by changing the scanning speed. To determine the frequency broadening of the LOFI signal caused by the scanning, we calculate the Fourier transform $\hat{S}(F)$ of the LOFI signal S(T). The modulus of $\hat{S}(F)$ is:

$$
\begin{aligned}
& |\hat{S}(F)| \propto G_{l o f i} \sqrt{R_{t}} \exp \left(-\frac{\pi\left(F-F_{d o p}-F_{e}\right)^{2}}{(2 \ell \dot{\alpha})^{2}}\right) \\
& \times \exp \left(\frac{\frac{2}{\pi W_{0}^{2}}}{\left(\frac{2}{\pi W_{0}^{2}}\right)^{2}+\left(\frac{2}{\lambda \ell}\right)^{2}}\right)
\end{aligned}
$$

For long distance imaging, the following condition occurs: $\left(\frac{2}{\pi W_{0}^{2}}\right)^{2}>\left(\frac{2}{\lambda \ell}\right)^{2}$ which corresponds to $1>>\mathrm{ZR}$, and the equation 7 becomes: 
$|\hat{S}(F)|^{2} \propto \exp \left(-\frac{\left(F-F_{d o p}-F_{e}\right)^{2}}{\Delta F_{d o p}{ }^{2}}\right)$

With $\Delta F_{d o p}=\frac{2 \ell}{\pi W_{0}} \dot{\alpha}$

\section{EXPERIMENTAL RESULTS AND VALIDATION}

For the experimental validation of the theoretical results, the distance between the moving mirror and the target is $\ell=3 \mathrm{~m}$. The target is a number plate which is constituted of black lettering on a retroreflective background. The waist of the laser beam focused on the target is $\mathrm{W}_{0}=230 \mu \mathrm{m}$. The frequency shift introduced by the AOMs is $\mathrm{F}_{\mathrm{e}}=2.4 \mathrm{MHz}$. The scanning system is mounted on the translation in order to control the misalignment $\Delta$. The mirror angular speed $(\dot{\alpha})$ is adjusted by changing the slope of the voltage ramp sent to the galvanometric mirror. The LOFI signal is measured by scanning a line of retro-reflectors on the target. Then the RF power spectrum is fitted with the theoretical curve given by equation 8 . The result obtained for a misalignment $\Delta=-10 \mathrm{~mm}$ and an angular speed $\dot{\alpha}=0.36 \mathrm{rad} . \mathrm{s}^{-1}$ is presented on figure 3 . The frequency shift and broadening calculated are $\mathrm{F}_{\mathrm{dop}}=-11.8 \mathrm{kHz}$ and $\Delta \mathrm{F}_{\mathrm{dop}}=2.1 \mathrm{kHz}$. The peak at $2.4 \mathrm{MHz}$ corresponds to parasitic light reflected between the AOM and the rotating mirror. All the light backscattered by the target is frequency shifted and corresponds to the peak centered at $2.388 \mathrm{MHz}$ (i.e. $\mathrm{F}_{\mathrm{e}}+\mathrm{F}_{\text {dop }}$ ). This spectrum shows that it is possible to filter the parasitic reflection by selecting the frequency $\mathrm{F}_{\mathrm{e}}+\mathrm{F}_{\mathrm{dop}}$ as a reference for lock-in detection.

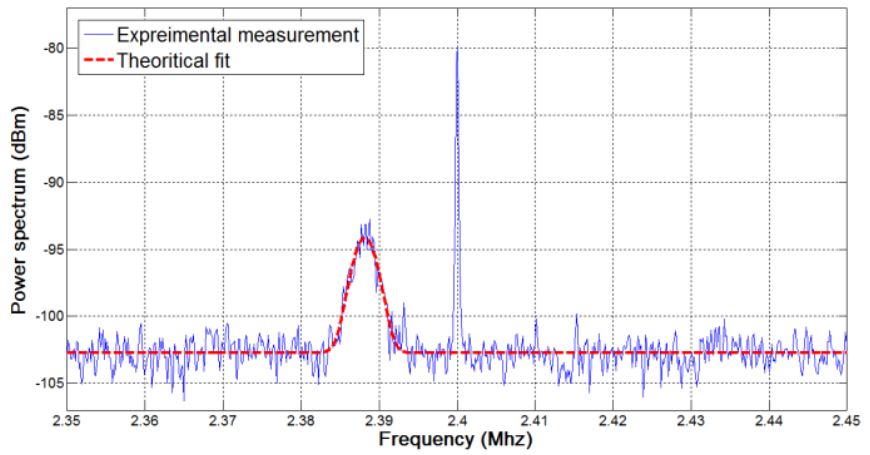

Figure 3: Experimental (solid curve) and theoretical (dashed curve) power spectra of the LOFI signal for a line scan. The target is a part of a number plate constituted of retroreflectors. The laser beam and the rotation axis of the moving mirror are misaligned. The broadband signal centered at 2.388 $\mathrm{MHz}$ corresponds to the target while the peak centered at $2.4 \mathrm{MHz}$ corresponds to parasitic reflected light.

To validate the theoretical results given by Eqs. 6 and 8, we have measured the Doppler frequency shift and the Doppler frequency broadening for different values of misalignment $\Delta$ and for different mirror angular speeds. The experimental results are presented in figure 4 and 5, one can observe a good agreement between the experimental and the theoretical results for both the frequency shift and the frequency broadening.

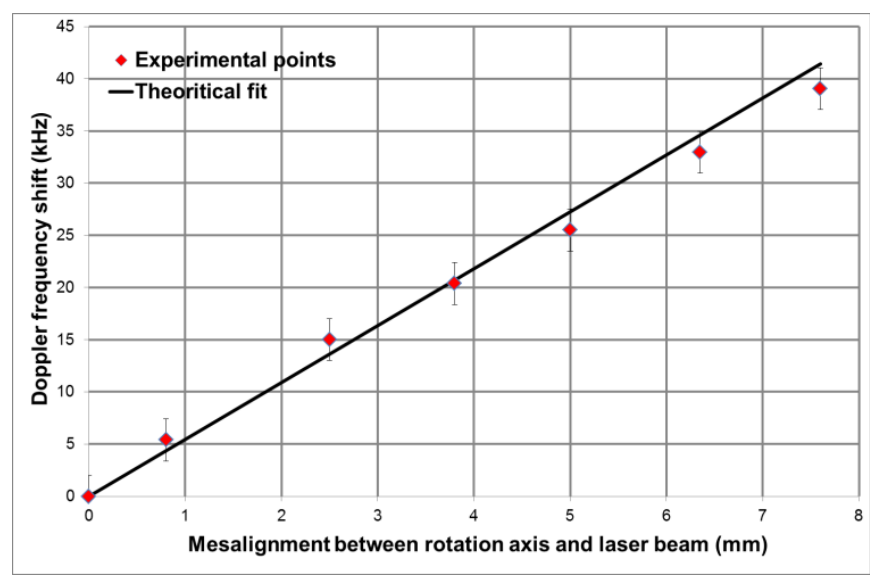

Figure 4: Evolution of the Doppler frequency shift $F_{\text {dop }}$ versus the misalignment $\Delta$. The dots correspond to the experimental points and the solid curve corresponds to equation 6 with $: \lambda=1.06 \mu \mathrm{m}$ and $\dot{\alpha}=0.36 \mathrm{rad} . \mathrm{s}^{-1}$.

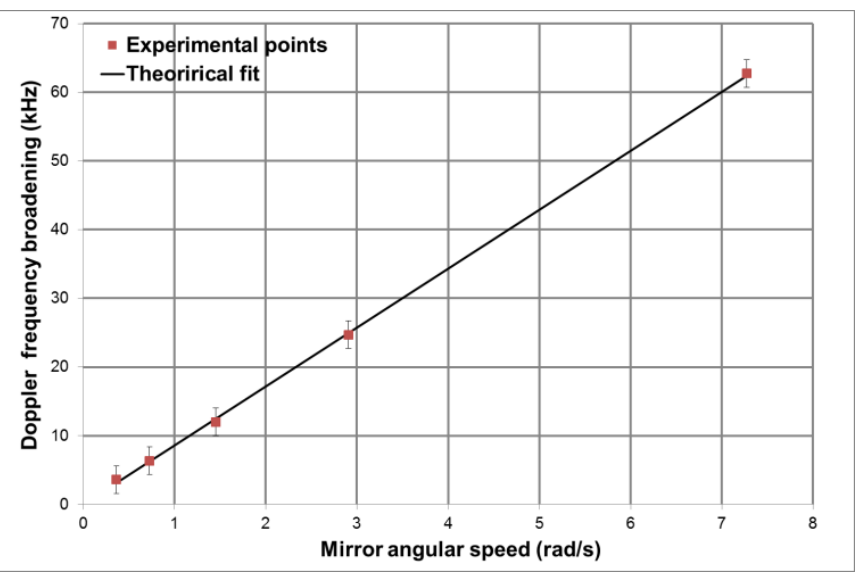

Figure 5: Evolution of the Doppler frequency broadening $\Delta \mathrm{F}_{\text {dop }}$ versus the mirror angular speed $\dot{\alpha}$. The dots correspond to the experimental points and the solid curve corresponds to equation 8 with $: \lambda=1.06 \mu \mathrm{m}$,

$\mathrm{W}_{0}=230 \mu \mathrm{m}$ and $\ell=3 \mathrm{~m}$.

\section{IMAGES SHOT NOISE LIMITED}

To confirm the possibility to filter the parasitic reflection contribution in LOFI images by using the Doppler effect induced by the moving mirror, we made images with and without Doppler shift with different reference frequencies $\mathrm{F}_{\text {ref }}$ for the lockin detection. The reference frequency is determined from the power spectra of the LOFI signal (Fig. 3) and it is generated with a function generator. For the image with the Doppler shift, the experimental conditions are $\Delta=-10 \mathrm{~mm}$ and $\dot{\alpha}=0.36 \mathrm{rad} . \mathrm{s}^{-1}$, the corresponding Doppler effects are presented on figure 3. For the image without the Doppler shift, the experimental conditions are $\Delta=0 \mathrm{~mm}$ and $\dot{\alpha}=0.36 \mathrm{rad} . \mathrm{s}^{-1}$. To simulate a scattering medium such as the fog, we have placed a tank filled with diluted milk. The corresponding losses are around-30dB for a round trip. The images are presented on figure 6 with the same greyscale levels so they can be compared. These images have been acquired in 4 $\mathrm{s}$ for a size of 200x200 pixels. This time corresponds to $100 \mu \mathrm{s}$ by pixel which is well suited with our instrumentation and gives mirror rotation speed $\dot{\alpha}=0.36 \mathrm{rad}_{\mathrm{s}} \mathrm{s}^{-1}$. We can consider three distinct areas in these images: The left area corresponds to the target and allows evaluation of the LOFI signals, while the right 
area without the target allows evaluation of the noise equivalent power (NEP) that is given by the sum of the laser quantum noise and the noise due to parasitic feedback. The horizontal dark band corresponds to the laser quantum noise. To obtain this band, the laser beam is temporally blocked between the frequency shifter and the beam splitter (figure 1) during the image acquisition, the signal measured is then the laser quantum noise at the frequency $\mathrm{F}_{\text {ref }}$ because no optical signal shifted in frequency is reinjected in the laser. If the level noise in a image is identical to LOFI signal in this dark band then the image is shot noise limited [10]. The figure 6 shows that the Doppler effect induced by the moving mirror coupled with the band pass filtering of the lock-in detection allows to considerably increase the contrast of LOFI images. In each image, an average value of the signal to noise equivalent power ratio (SNR) has been calculated. In the figure 6(a) the SNR is equal to 45 while in the figure 6(b) the $\mathrm{SNR}$ is equal to 3 . The NEP in the figure 6(b) is much greater than the quantum noise of the laser, the ratio between the NEP and the quantum noise of the laser is around 80 (average on 625 pixels). In contrast, the image 6(a) presents a NEP equivalent to quantum noise of the laser (the ratio is equal to 1.01), i.e. the image is therefore shot noise limited.

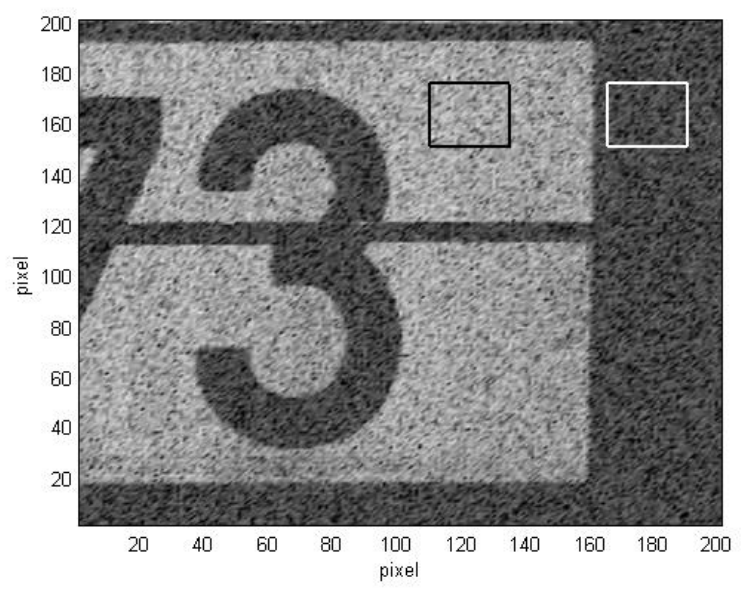

(a)

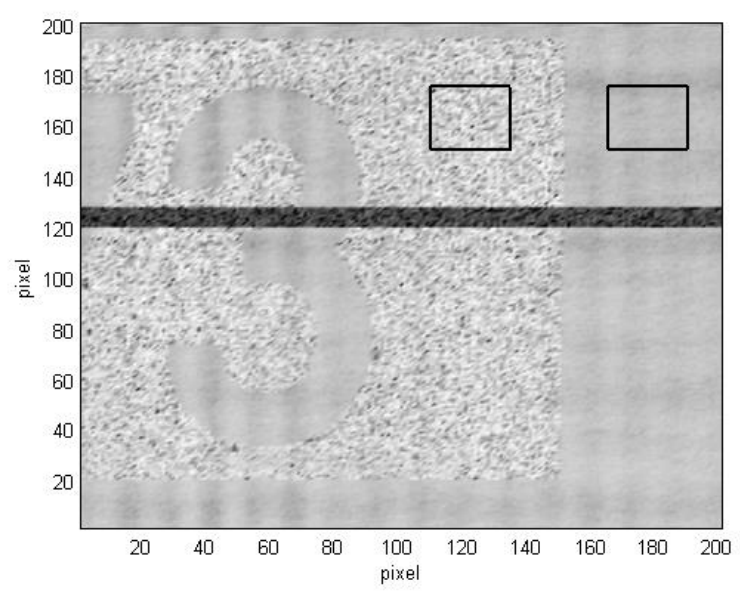

(b)

Figure 6: Images of a number plate through a scattering medium: (a) the reference frequency for the lock-in detection is $\mathrm{F}_{\text {ref }}=\mathrm{F}_{\mathrm{e}}-\mathrm{F}_{\text {dop }}=2.3882 \mathrm{MHz}$, (b) the reference frequency for the lock-in detection is $\mathrm{F}_{\text {ref }}=\mathrm{F}_{\mathrm{e}}=2.4 \mathrm{MHz}$. The left (resp. right) rectangle represents the area where the signal (resp. $\mathrm{NEP}$ ) is measured. Both rectangles contain 625 pixels.

\section{CONCLUSION}

We have demonstrated the possibility to filter the parasitic feedback effect in LOFI image by using a frequency Doppler shift induced by the mirror motion during the target scanning. Unlike previously proposed methods, this very simple filtering is well adapted for a distant target and it considerably enhances the contrast and therefore the sensitivity of the LOFI technique. The dependence of the frequency shift and the frequency broadening of the LOFI signal caused by the scanning of the punctual target were theoretically demonstrated and were experimentally verified. We have also demonstrated the possibility to obtain shot noise limited images by this way. The detection limit is then given by the quantum noise of the laser. It is important to note that for a good filtering, the scanner must be placed after all the other optical components of the setup which is not necessarily well suited for a very close target. Another limitation of the Doppler filtering is the difficulty to realize phase imaging because it is difficult to perfectly adjust the reference frequency of the lock-in on the LOFI signal frequency. In a near future, we would like to test this setup in real conditions to realize amplitude images of targets located twenty meters through fogs with different visibilities to quantify the performances and possible future applications.

\section{References}

1. J. M. Schmitt, A. Knüttel, and M. Yadlowsky "Confocal microscopy in turbid media" J. Opt. Soc. Am. A 11, 22262235 (1994).

2. C. Dunsby and P. M. W. French "Techniques for depthresolved imaging through turbid media including coherence-gated imaging” J. Phys. D: Appl. Phys. 36, R207 (2003)

3. J.M. Schmitt and A. Knüttel, "Model of optical coherence tomography of homogeneous tissue" J. Opt. Soc. Am. A14, 1231-1242 (1997).

4. O. Jacquin, E. Lacot, W. Glastre, O. Hugon, and H. Guillet de Chatellus, "Experimental comparison of autodyne and heterodyne laser interferometry using Nd:YVO4 microchip laser," J. Opt. Soc. Am. A, vol. 28, 1741-1746, (2011).

5. K. Otsuka, "Effects of external perturbations on LiNdP4O12 lasers, ” IEEE J. Quantum Electron. 15, 655 663 (1979).

6. E. Lacot, R. Day, and F. Stoeckel, "Coherent laser detection by frequency-shifted optical feedback," Phys. Rev. A, vol. 64, pp. 043815(1-11), (2001).

7. K. Otsuka, "Self-Mixing Thin-Slice Solid-State Laser Metrology," Sensors, vol. 11, 2195-2245, (2011).

8. S. Suddo, T. Ohtomo, Y. Takahascvhi, T. Oishi, and K. Otsuka, "Determination of velocity of self-mobile phytoplankton using a self thin-slice solid-state laser", Appl. Opt. 48, 4049 - 4055 (2009).

9. H. Gilles, S. Girard, M. Laroche, and A. Belarouci, "Nearfield amplitude and phase measurements using heterodyne optical feedback on solid-state lasers", Opt. Lett. 33, 1 - 3 (2008).

10. W. Glastre, O. Jacquin, O. Hugon, H. Guillet de Chatellus, and E. Lacot, "Deep and optically resolved imaging through scattering media by space-reversed propagation", Opt. Lett., vol. 37, pp. 4825-4823, (2012). 
11. O. Jacquin, S. Heidmann, E. Lacot and O. Hugon," Selfaligned setup for laser optical feedback imaging insensitive to parasitic optical feedback", Applied Optics, Vol. 48, No.1, 64-68 (2009).

12. O. Jacquin ,W. Glastre, E. Lacot, O. Hugon, H. Guillet de Chatellus, F. Ramaz "Acousto-optic laser optical feedback imaging," Opt. Lett., vol. 37, pp. 2514-2516, (2012).

13. W. Glastre, O. Jacquin, O. Hugon, H. Guillet de Chatellus, E. Lacot, Synthetic aperture laser optical feedback imaging using a translational scanning with galvanometric mirrors, J. Opt. Soc. Am. A. 29, 1639 (2012) 